\title{
Labyrinthe
}

22 | 2005 (3)

La Biopolitique (d')après Michel Foucault

\section{Individuation et pouvoir politique}

Une lecture de Foucault et d'Elias

\section{Emmanuel Taïeb}

\section{(2) OpenEdition \\ Journals}

Édition électronique

URL : http://journals.openedition.org/labyrinthe/1032

DOI : $10.4000 /$ labyrinthe.1032

ISSN : 1950-6031

Éditeur

Hermann

\section{Édition imprimée}

Date de publication : 1 novembre 2005

Pagination : 37-46

\section{Référence électronique}

Emmanuel Taïeb, « Individuation et pouvoir politique », Labyrinthe [En ligne], 22 | 2005 (3), mis en ligne le 22 juillet 2008, consulté le 19 avril 2019. URL : http://journals.openedition.org/labyrinthe/1032

DOI : $10.4000 /$ labyrinthe.1032

Propriété intellectuelle 


\title{
INDIVIDUATION \\ ET POUVOIR POLITIQUE \\ Une lecture de Foucault et d'Elias
}

\author{
Emmanuel TAÏ̈B \\ etaieb@club-internet.fr
}

S'il y a une lacune que la publication de Sécurité, territoire, population et Naissance de la biopolitique, de Foucault, fait apparaître chez ses continuateurs se réclamant notamment du biopouvoir, c'est bien la question de l'individu, et de la place qui lui est réservée dans la pensée du pouvoir politique. Les modèles proposés par Agamben ou Sloterdijk restent dominés par un holisme méthodologique qui se marque par une inattention à l'endroit de l'individu et de la subjectivation ${ }^{1}$, ou qui ne considère l'individu que comme un corps biologique, devenu modalité du pouvoir et son lieu d'exercice premier.

Le point envisagé ici n'est ni celui de l'apparition d'une subjectivation politique, ni celui d'une liberté qui serait produite par le pouvoir $\rightarrow$ voir l'article de D. Sardinha $\leftrightarrow$, mais d'une individuation permise et produite par des formes particulières de pouvoir. Dans les travaux de Foucault, ces formes de pouvoir ressortissent du pouvoir pastoral, lequel entretient avec la notion de «police» des affinités marquées, dans leur gestion commune du groupe humain, et dans la volonté de le connaître pour garantir sa survie et son amélioration; et de la notion désormais sursignifiante de biopouvoir, entendue non comme mode d'exercice dominant de la souveraineté, ni comme vitalisme ontologique permettant la contestation de l'ordre politique $\leadsto$ voir l'article d'A. Skornicki $\hookrightarrow$, mais plutôt comprise comme administration politique de l'être-corps des composantes de la population ${ }^{2}$.

\footnotetext{
1. Que l'on considère notamment la perspective juridico-institutionnelle d'Agamben dans État d'exception. Homo sacer, II, 1, Paris, Seuil, coll. «L'ordre philosophique », 2003.

2. Sur les différentes acceptions du biopouvoir, voir Jacques Rancière, «Biopolitique ou politique? », Multitudes, 1, mars 2000.
} 
L'existence historique du pouvoir pastoral, de l'État de police, comme la présence contemporaine de formes de biopouvoir, permettent aux analyses de Foucault d'injecter dans une perspective originellement holistique la question de l'état du pouvoir dans son capillaire ultime. Dans la société disciplinaire, le corps fait retour, en ce qu'il accueille la punition du souverain. Dans le pouvoir pastoral, l'État berger a la capacité, et la préoccupation, de passer par le niveau de sa plus petite composante pour assurer la survie du troupeau. Dans le biopouvoir, la gestion de l'être-corps implique une attention croissante à ses états pour mieux le contrôler et le faire vivre. Pour autant, Foucault n'insiste pas sur les conditions d'individuation rendues possibles par ces formes de souveraineté, et ce n'est qu'au prix d'un détour par l'Antiquité qu'il s'intéressera au « souci de soi $^{3} »$.

La sociologie de Norbert Elias a, elle, toujours tenté de faire tenir ensemble holisme et individualisme, en reliant la formation et la forme de l'État à l'économie psychique individuellet. À côté du processus de civilisation se place un processus d'individuation, qui se marque par l'intériorisation de contraintes externes par les sujets, donc d'un autocontrôle individuel qui renforce la conscience de soi et permet, potentiellement, le développement d'une individualités, ou d'une subjectivation politique, hors du groupe. L'individuation permet aussi l'identification d'un soi contrôlé et des autres comme se contrôlant. Produite par les contraintes étatiques et la configuration sociale changeante, l'individuation en est aussi la condition.

Le pouvoir, chez Foucault et Elias, gère de la multiplicité, mais fabrique de l'individuel. L'individuation est un effet émergent des différents modes de gouvernement, non pas parce que dans une perspective d'individualisme méthodologique l'individu serait toujours premier, mais parce que l'individu est à la fois ce qui est produit par le pouvoir, son lieu d'exercice, mais aussi ce qui résiste au pouvoir.

3. Histoire de la sexualité. III. Le souci de soi, Paris, Gallimard, coll. « Tel », 2004 [1984].

4. Pour des informations biographiques mises en perspective, autour d'un Elias traversant le $\mathrm{XX}^{\mathrm{e}}$ siècle (1897-1990), tout à la fois exilé d'Allemagne, Juif dont la mère périra à Auschwitz, enseignant marginal à la carrière chaotique, mais dont l'apport sociologique est le produit de cette même trajectoire, voir Bernard Lacroix, « Portrait sociologique de l'auteur », dans Alain Garrigou, Bernard Lacroix (dir.), Norbert Elias. La politique et l' histoire, Paris, La découverte, 1997.

5. Norbert Elias, La Société des individus, Paris, Fayard, coll. «Pocket Agora », 1997, p. 101. 


\section{L'individu en souveraineté}

Sous l'aspect de l'individuation, les rapprochements entre les analyses de Foucault et d'Elias sont nombreux ${ }^{6}$. La notion de discipline dégagée par Foucault trouve un équivalent fonctionnel dans la notion eliassienne d'accroissement des chaînes d'interdépendance entre les individus, dans les sociétés à monopole de la violence physique légitime par une unité étatique centrale $\mathrm{e}^{7}$ Ce phénomène typique de la modernité, emprunte à la fois à une division poussée du travail, et à une spécialisation-technicisation des tâches qui rend les individus de plus en plus dépendants les uns des autres pour l'accomplissement des actes de la vie quotidienne et la satisfaction des besoins élémentaires. Monopole et chaînes d'interdépendances forment ensemble une configuration sociale particulière qui pèse sur les individus, à laquelle ils participent et se conforment. La discipline foucaldienne comme la configuration eliassienne conduisent à des changements dans le rapport à soi. L'individuation disciplinaire produit une discipline du moi capable de le rendre conforme à l'idéal bureaucratique ou politique, tandis que l'individuation conditionnée par la configuration concourt à une maîtrise de soi en société, et à la conformation à des modèles ouvrant à la vie en commun, à la vie curiale ou à la vie démocratique. Discipline et configuration participent donc également d'une autogestion des individus, et d'une contrainte des désirs et des pulsions corporels qui mettent fin à la violence interpersonnelle et participent d'une «sociétalisation du $\mathrm{moi}^{8} \gg$.

Que la discipline passe par des institutions identifiées, chez Foucault, ou qu'elle passe par les mutations du monopole central, chez Elias, dans les deux hypothèses l'objectif politique est de fabriquer des corps dociles, conformés, et d'obtenir un contrôle de la vie individuelle. Lequel produit en retour les phénomènes d'individuation, via un contrôle de l'économie

\footnotetext{
6. Robert van Krieken, « The Organization of the Soul : Elias and Foucault on Discipline and the Self », Archives européennes de sociologie, tome XXXI, 2, 1990; Numa Murard, « Norbert Elias et Michel Foucault: deux flèches au cœur de la subjectivité », Tumultes, 15, octobre 2000.

7. Selon Pieter Spierenburg, les deux auteurs ont aussi en commun la vision d'un pouvoir omniprésent, qui ne saurait se réduire à un centre ou à un individu, voir « Punishment, Power, and History. Foucault and Elias », Social Science History, vol. 28, 4, Winter 2004, p. 625.

8. Robert van Krieken, « The Organization of the Soul... », art. cit., p. 355.
} 
psychique personnelle, avec les dispositifs de confession ou d'aveu ${ }^{9}$, avec l'inculcation des «bonnes manières » et de modèles d'idéal du moi social, qui donnent à l'individu le sentiment poussé d'un soi unique dans le groupe, et d'une intériorité façonnable et contrôlable pour évoluer au mieux dans ce groupe et pour atteindre l'idéal politique du moi.

Foucault comme Elias ont mis en évidence que la gouvernementalité était avant tout un gouvernement des conduites, une conduite des conduites, et que ce que cherche à obtenir le pouvoir, c'est un autocontrôle des individus, par le recours à la normalisation, chez Foucault, ou aux civilités, chez Elias ${ }^{10}$. Normalisation et autocontrôle individuel visent tous deux à obtenir une pacification interne de la société et une intériorisation des contraintes étatiques ou externes, qui s'achèvent en un retrait relatif de l'État sur ses fonctions régaliennes, au profit d'une prise en charge de nombre de ses fonctions par les individus. Bien que pris dans des formes de souveraineté, l'individu n'est jamais absent. Les sociétés disciplinaires, de contrôle et de normalisation entendent contrôler sa subjectivité, et les sociétés issues du processus de civilisation entendent lui confier individuellement ce contrôle, afin d'en garantir la meilleure application et de faire concorder individuation et idéal politique du moi.

\section{Le pouvoir pastoral, matrice de l'individuation}

En proposant le modèle du pouvoir pastoral (STP, p. 126), Foucault livre en réalité plus qu'un modèle historiquement situé. En étendant le système pastoral au processus d'individuation - usage que Foucault mentionne seulement, parlant d'un «pouvoir individualisateur ${ }^{11} »-$, celui-ci apparaît comme une matrice qui contient et influence les modèles de pouvoir qui naîtront plus tardivement.

\footnotetext{
9. L'aveu du criminel fait l'objet de développements dans Surveiller et Punir. Naissance de la prison, de Foucault, Paris, Gallimard, coll. « Tel », 1993 [1975], p. 48 sqq.

10. Norbert Elias, La Civilisation des moeurs, Paris, Calmann-Lévy, coll. «Pocket Agora », 2000 [1939]; Id., La Dynamique de l'Occident, Paris, Calmann-Lévy, coll. « Pocket Agora », 1996 [1939].

11. " "Omnes et singulatim": vers une critique de la raison politique » (1979), dans Dits et Écrits, IV, Paris, Gallimard, coll. « Bibliothèque des sciences humaines », 1994, p. 136.
} 


\section{Éléments du gouvernement pastoral}

Forme idéal-typique de régime, qui trouve son origine dans les sociétés antiques d'Orient (Égypte, Assyrie, Judée), le pouvoir pastoral est un décalque politique des relations entre Dieu et les hommes. Un roi-berger surveille et gère un peuple-troupeau, dont il doit assurer la subsistance, les soins, et le salut. Dans la conception hébraïque, la relation pasteur-brebis est d'obéissance, alors que dans l'appropriation chrétienne du pastorat, elle est de dépendance et de soumission individuelle. Obéissance ou soumission deviennent des fins en soi, où la responsabilité du pasteur est engagée, et où le péché de chaque membre du troupeau est imputable à son chef. D'où une nécessaire connaissance que le pasteur doit posséder de son troupeau, laquelle, en retour, autorise l'individualisation. Le pasteur doit être attentif à chaque membre du groupe, et finalement entretenir un rapport individualisé à la multiplicité de ses brebis. Pour accéder à ce savoir, le christianisme a importé puis développé deux outils helléniques: l'examen de conscience, qui permet la maîtrise de soi, et la direction de conscience, qui permet la conduite des hommes dans leur vie quotidienne.

Ce modèle ne disparaît pas lors de la formation des États occidentaux, et irrigue les théories de la raison d'État et de la "police», qui s'intéresse à la population vivante en tant que son bien-être permettrait de renforcer la puissance de l'État.

Le pouvoir pastoral annonce le pouvoir de souveraineté en autorisant l'existence d'une modalité individuelle. Car, pour gérer du nombre, le pouvoir doit prendre en compte les existences individuelles des membres composant le groupe. Le «troupeau » humain reste pensé dans une perspective organiciste, comme un corps qu'il faut préserver dans sa totalité, soit en sauvant un membre gâté, soit en le supprimant (STP, p. 172). C'est ici que le droit du souverain de réclamer, ordonner, et procéder à la mise à mort d'un de ses citoyens trouve sa source. Selon Foucault, le crime 
puni de mort est avant tout un crime contre la souveraineté ${ }^{12}$, c'est-à-dire un crime contre le corps collectif contractualiste. Quand au XIX $X^{\mathrm{e}}$ siècle triomphe la théorie de la défense sociale ${ }^{13}$ pour justifier la peine de mort, elle ne se rend compréhensible qu'en filant à son tour la métaphore organologique et en légitimant l'élimination physique des criminels par des considérations hygiénistes et de purification du corps social, assimilables finalement à des théories pastorales renouvelées. Le pouvoir pastoral autorise donc la suppression du membre criminogène gangrené, pour la survie du groupe, tout comme le feront les sociétés de souveraineté.

Mais en frappant un condamné pris isolément, le souverain contribue à inventer l'individualisation de la peine. Pas dans ses formes contemporaines d'une souplesse du droit selon la personnalité de l'accusé, mais dans l'habituation à des peines qui ne s'exercent que sur un corps à la fois. Et les passages que consacre Foucault, dans Surveiller et Punir, au supplice de Damiens en 1757, marquent tout autant la fin des punitions d'Ancien Régime et la voie vers l'égalité révolutionnaire de la guillotine, que le moment où la peine choque, parce qu'elle marque la démesure entre la puissance du souverain et le corps dominé du condamné sur qui s'exerce cette puissance. Dans les sociétés de souveraineté, et dans les sociétés disciplinaires, la peine est pensée et perçue comme ce qui s'applique au seul corps individualisé d'un condamné, connu, nommé, identifié. Et les difficultés d'acclimatation de la prison, assimilée à une peine collective, prenant en charge des masses de condamnés anonymes, et les dissimulant au regard, expliquent la répugnance à légiférer sur les formes de l'incarcération ${ }^{14}$, et les mouvements réclamant le maintien de la peine de mort ${ }^{15}$, voire le retour des châtiments corporels; c'est-à-dire des peines visibles et impliquant un condamné donné.

Le droit de punir, dans les approches proposées par Elias, change au moment du passage aux sociétés de souveraineté, c'est-à-dire quand le monopole de la violence physique par une puissance centrale est achevé. Cette monopolisation se marque par l'appropriation, par le souverain, des

12. Les Anormaux. Cours au Collège de France, 1974-1975, Paris, Gallimard/Seuil, coll. «Hautes études», 1999, p. 76; Foucault, SP, p. 107.

13. Martine Kaluszynski, La République à l'épreuve du crime. La construction du crime comme objet politique. 1880-1920, Paris, LGDJ, MSH-RED\&S, coll. « Droit et société », 2002.

14. Robert Badinter, La Prison républicaine (1870-1914), Paris, Fayard, coll. « Le Livre de poche », 1992.

15. Julie Le Quang Sang, «L'abolition de la peine de mort en France: le rendez-vous manqué de 19061908 », Crime, Histoire \& Sociétés, vol. 6, 1, 2002. 


\section{Individuation et pouvoir politique}

moyens de la justice, qui de sociétale et privée, devient exclusivement publique, et tendant à sa nationalisation, via une homogénéisation du droit et sa codification. Monopolisation qui se marque aussi par l'institutionnalisation puis la professionnalisation du bourreau, au XVI ${ }^{e}$ siècle, selon Pieter Spierenburg ${ }^{16}$ qui se situe dans la perspective de Norbert Elias.

Le pouvoir pastoral annonce, négativement, le pouvoir disciplinaire, par le biais de l'ascétisme comme contre-conduite (STP, p. 209). L'ascétisme apparaît comme un corps à corps avec soi-même, sans l'autorité d'un autre, et surtout donc hors de l'autorité du pasteur. Il est un défi interne, lancé à soi-même, à son corps, et un défi externe à destination de l'autorité souveraine. Selon Foucault, l'ascétisme est une obéissance retournée devenue maîtrise de soi (STP, p. 209-211), et sous cet aspect une forme de résistance individuelle, passant par la discipline individuelle, au pouvoir pastoral. Mais là ou Foucault généralise cette contreconduite au sein du pouvoir pastoral comme fondement du « rêve disciplinaire » $(S T P$, p. 348) qui irrigue le bon gouvernement de l'État, la «police» $(S T P$, p. 321), Elias individualise la pratique ascétique. La maîtrise de soi provient de la configuration sociale, mais ne lui confère pas sa forme, et reste limitée à un autocontrôle individuel. L'autocontrôle ne vise d'ailleurs pas en priorité la maîtrise des pulsions, sexuelles ou alimentaires, il ne vise pas la discipline pour la discipline, mais il vise la maîtrise de soi comme discipline sociale. C'est-à-dire comme élément déterminant dans le « jeu » social propre à une configuration, où il s'agit de ne pas montrer ses sentiments, ses émotions, de ne pas perdre la face, dans le lexique de Goffman, de ne pas recourir à la violence, donc de se contrôler pour contrôler sa position dans les rapports de force qui engagent l'individu ${ }^{17}$. La discipline de soi, c'est la transformation du corps contrôlé en outil politique, au service de la « survie » sociale et politique de l'individu.

Foucault pose donc une conduite disciplinaire individuelle devenue modèle social, tandis qu'Elias pose un modèle de société impliquant l'autocontrainte individuelle. Foucault en trouve la source dans un pouvoir pastoral qui oblige à l'obéissance, sans la discipline, là où Elias

16. The Spectacle of Suffering. Executions and the Evolution of Repression: from a Preindustrial Metropolis to the European Experience, Cambridge, Cambridge University Press, 1984, p. 25.

17. Norbert Elias, La Société de cour, Paris, Flammarion, coll. « Champs », 1995 [1969]; Id., « On transformation of aggressiveness », Theory and Society, vol. 5, 2, mars, 1978. 
en trouve la source dans des modèles déjà disciplinaires, impliquant obéissance et discipline, donc ressortissant à la fois du pouvoir pastoral et du normatif disciplinaire. Le monastère et l'armée ont été souvent identifiés comme les lieux d'invention de ce modèle disciplinaire ${ }^{18}$. Ces institutions totales nécessitent une complète égalité de leurs membres, c'est-à-dire l'effacement de leurs particularités individuelles, pour la gestion optimale du groupe et le rationnement de ses besoins (pastorat) et pour la gestion d'individus identiques, soumis à une hiérarchie de commandement, ayant intériorisé les règles (discipline). Le monastère et l'armée ont donc, les premiers, inventé la « police » et la gestion d'une population captive, tout entière tournée vers des buts transcendants l'individu, mais pour lesquels il doit être en parfaite condition physique. Quand Bruno Karsenti écrit que la discipline appliquée au social, c'est la perspective militaire convertie en perspective éducative ${ }^{19}$, il faudrait compléter, avec Elias, pour dire que l'éducation de soi, c'est la perspective disciplinaire convertie en outil et condition du jeu politique, et en possibilité de vivre en société. Le paradoxe veut donc qu'une culture individualiste se développe dans les lieux mêmes de la culture groupale et de la rationalisation des corps.

\section{Le corps du biopouvoir}

C'est ce qu'annonce finalement le pouvoir pastoral dans sa préoccupation partagée avec la « police » d'un bien-être collectif: un biopouvoir tourné vers la santé du corps, et vers une « bioréflexivité20 ${ }^{2}$, une incorporation individuelle du biopouvoir étatico-médical par le truchement de la maîtrise de son corps pulsionnel et morbide. Le biopouvoir, tel que l'entend par exemple Agamben, comme une forme nouvelle de répression axée sur le contrôle de l'être-corps des citoyens, n'a pas de sens chez Elias, pour qui le pouvoir politique est toujours-déjà un pouvoir sur les corps, un pouvoir avec les corps. L'absence de partage possible entre le

18. Bryan S. Turner, « The Rationalization of the Body: Reflections on Modernity and Discipline », dans Scott Lash, Sam Whimster (eds), Max Weber, Rationality and Modernity, London, Allen \& Unwin, 1987.

19. « Le criminel, le patriote, le citoyen. Une généalogie de l'idée de discipline », L'Inactuel, « L'idée de meurtre », 2, printemps 1999, p. 125.

20. Dominique Memmi, Faire vivre et laisser mourir. Le gouvernement contemporain de la naissance et de la mort, Paris, La Découverte, coll. « textes à l'appui/politique et société », 2003, p. 202. 
corps biologique et le corps politique, où Agamben fait sourdre le paradigme biopolitique de la modernité ${ }^{1}$, n'existe pas chez Elias où le corps du politique est le corps biologique individuel, et où le corps biologique est éminemment politique. Elias rejoint ici le Foucault du pouvoir pastoral et de ses successeurs, pour affirmer que le pouvoir politique a, dès l'origine, géré des corps.

Ce pouvoir politique, soucieux de sa population, soucieux du devenir de ses corps, soucieux du « faire vivre » plutôt que du «faire mourir », est un biopouvoir « bienfaisant» (STP, p. 130), dont on retrouve l'écho dans le biopouvoir médical contemporain. Il reste bien sûr possible de soutenir que le biopouvoir médical est en dernière instance une forme de normalisation, et un gouvernement de l'espèce humaine par le biais de l'administration de la vie. Et un usage du terme dénonce d'ailleurs une approche technocratique où le pouvoir est confié à des experts au nom d'impératifs de santé publique, et où le contrôle du vivant fabriquerait, via les perspectives de clonage humain, d'eugénisme, ou d'euthanasie, un être-corps humain qui ne s'appartiendrait plus ${ }^{22}$.

Cependant, si l'on tient ce biopouvoir médical comme désireux avant tout du «faire vivre », sans autre considération que le refus de la mort et de la maladie, si l'on conçoit le pouvoir politique comme devant s'assurer de la sécurité corporelle de ses citoyens, les formes du biopouvoir médical l'apparentent alors à un gouvernement des conduites. Dominique Memmi a mis en évidence que ce biopouvoir médical était devenu un biopouvoir délégué à l'individu ${ }^{23}$, en ce sens que l'État se déleste du contrôle médical des corps, au profit d'un contrôle de soi individualisé. On passe sensiblement d'un pouvoir disciplinaire à un pouvoir où la contrainte externe est affaiblie, au profit d'une autocontrainte, qui est simplement accompagnée par l'État et vérifiée par le médecin. On passe d'une « institution disciplinaire » foucaldienne à une « institution civilisatrice » eliassienne, où chaque citoyen est appelé à connaître les maladies, à s'en protéger, à maîtriser ses pulsions sexuelles, à utiliser des moyens de contraception ou de protection contre les maladies infec-

21. Homo Sacer. Le pouvoir souverain et la vie nue, Paris, Seuil, coll. « L'ordre philosophique », 1997 [1995], p. 202.

22. Virginie Tournay, « Le biopouvoir à l'épreuve des travaux sur la biomédecine: succès politique d'un néologisme », dans Sylvain Meyet, Marie-Cécile Naves, Thomas Ribemont (dir.), Travailler avec Foucault. Retours sur le politique, Paris, L'Harmattan, coll. « Cahiers politiques », 2005.

23. Faire vivre et laisser mourir..., op. cit., p. 292. 
tieuses. C'est sur lui que repose en fin de compte la gestion de sa propre santé, par le contrôle de son être-corps. Cette valorisation de l'autocontrôle est une marque du progrès de l'individuation et incarne l'avènement d'un sujet triomphant, pensé comme capable d'anticipation et de rationalisation dans les usages qu'il peut faire de son corps. Elle n'est pensable que dans l'entérinement que le processus de civilisation s'est accompagné d'un processus d'individuation, qui permet le retrait de l'État quand l'individu montre qu'il est capable de se contrôler. Le biopouvoir étatique a vocation à disparaître dès qu'il aura été incorporé au niveau individuel. Il cesse d'être contrainte quand il devient autocontrainte. Dès lors, le corps biologique et le corps politique s'indifférencient au sein de l'être-corps individuel.

Le souci de soi, alors, n'appartient pas à une époque en particulier, il est un effet de l'individuation produite par les diverses figures du pouvoir et par la place laissée toujours libre à l'individu au sein des dispositifs étatiques. Pour Foucault, l'individu est ce qui résiste au pouvoir, et chez Elias il est son auxiliaire, celui qui permet à la forme étatique de se maintenir, une fois qu'elle a été individuellement intériorisée. Le pouvoir s'abîme dans l'individu, avec pour objectif de devenir une contrainte interne seulement et de disparaître comme contrainte externe. L'État devient un simple gestionnaire des besoins vitaux, dans une société d'individus qui peuvent vivre hors de son contrôle permanent. Le pouvoir est donc toujours dans l'utopie de sa propre disparition: par le contentement des besoins, et par l'autocontrôle, il dépasse les classes sociales et leurs conflits, pour ne gérer que des corps vivants, sans recourir à la violence. Le social réinventé, et la résistance au (bio)pouvoir, passent alors par la garantie de l'individuation, plus que par la multitude. Et l'individu demeure.

Le rapprochement entre les perspectives de Foucault et celles d'Elias a mis en exergue la prégnance de l'individuation dans les manifestations et les ambitions du pouvoir politique. Et ce n'est pas que théorique, car le Foucault du souci de soi avait redécouvert l'importance conceptuelle de l'individuation, et à la fin de sa vie traduisait, pour son propre plaisir, La Solitude des mourants, ouvrage tardif de Norbert Elias ${ }^{24}$.

24. Stephen Mennell, «Beyond Elias », Figurations. Newsletter of the Norbert Elias Foundation, 1, juin 1994. Cette information est donnée dans la chronologie ouvrant les Dits et Écrits. 